\title{
NOUVELle
}

\section{In bacteria veritas}

\section{Le rôle pronostique du microbiote intestinal dans le cancer}

Meriem Messaoudene ${ }^{1}$, Lisa Derosa ${ }^{2,3}$, Wiam Belkaid ${ }^{1}$, Bertrand Routy ${ }^{1,4}$

> L'immunosurveillance, qui consiste en la reconnaissance et la destruction des cellules cancéreuses par le système immunitaire, a considérablement été intégrée ces dernières années dans la prise en charge des patients atteints de cancer avec le développement des immunothérapies. Depuis 2011, l'apparition d'une nouvelle classe d'immunothérapie, les inhibiteurs des points de contrôles immunitaires ( $\mathrm{ICl}$ ), a en effet révolutionné les traitements contre le cancer [1].

Les points de contrôles immunitaires protègent les tissus contre les dommages lorsque le système immunitaire réagit aux agents pathogènes et préviennent une réaction non contrôlée menant vers une auto-immunité. Cette régulation des fonctions effectrices s'opère via l'expression par les lymphocytes T (LT) de molécules telles que CTLA-4 (cytotoxic T-lymphocyte-associated antigen 4) et PD-1 (programmed death-1), dont les ligands respectifs sont B7 (contrerécepteur $\mathrm{CD} 80$ ) et $\mathrm{PD}-\mathrm{Ll} / 2$ (programmed death ligand-1/2). Un nombre croissant de preuves démontre qu'un des mécanismes primaires par lequel les tumeurs échappent au système immunitaire serait l'engagement de ces points de contrôle.

Afin de réactiver les réponses antitumorales, et de contourner ces mécanismes d'échappement, les $\mathrm{ICl}$ tels que les anticorps monoclonaux anti-PD-1 et anti-CTLA-4 ont été ainsi développés et approuvés dans le traitement de nombreux cancers incluant le mélanome, les cancers du poumon et du rein.
Ces anticorps, initialement proposés en deuxième ligne, progressent rapidement dans la prise en charge des patients et sont administrés en première ligne ; ils le seront prochainement en néo-adjuvant pour les cancers limités [2, 3].

Malgré l'efficacité clinique des $\mathrm{ICI}$, chez plus de $70 \%$ des patients, la maladie continue de progresser, et ces traitements entraînent de nouveaux effets secondaires de type auto-immun tels que des colites, des pneumonites et du vitiligo. Un des objectifs actuels en immuno-oncologie est ainsi d'étudier les mécanismes expliquant ces résistances et de développer de nouveaux biomarqueurs afin de prédire la réponse clinique [1].

De manière inattendue, plusieurs études, dont celles de notre groupe, ont récemment identifié que le microbiote intestinal pourrait être en partie responsable des réponses immunitaires anti-tumorales des $\mathrm{ICl}$.

Le microbiote intestinal, regroupant plus de $10^{13}$ bactéries, archeae, champignons et virus, est de plus en plus reconnu comme ayant des rôles fondamentaux en physiologie et en santé humaine. Sa composition est façonnée par plusieurs éléments qui ne sont pas uniquement liés à des facteurs génétiques, mais également à des facteurs environnementaux, liés au régime alimentaire, à la prise de médicaments et aux mesures anthropométriques ${ }^{1}$, et représentant

\footnotetext{
${ }^{1}$ Les mesures anthropométriques permettent d'évaluer la corpulence, les proportions et la composition du corps humain. Elles sont le reflet de l'état nutritionnel et de santé de la personne.
}

${ }^{1}$ Centre de recherche du centre hospitalier de l'université de Montréal (CRCHUM), 900 rue Saint-Denis, H2X 3 H8 Montréal, Québec, Canada.

${ }^{2}$ Campus cancer Gustave Roussy, Inserm U1015, Villejuif. Équipe labellisée-Ligue nationale contre le cancer, Villejuif, France.

${ }^{3}$ Université Paris-sud, université Paris-

Saclay, Gustave Roussy, Villejuif, France.

${ }^{4}$ Division d'hémato-oncologie, département de médicine, centre hospitalier de l'université de Montréal (CHUM), Montréal, Québec, Canada.

meriem.messaoudene@umontreal.ca bertrand.routy@umontreal.ca

plus de $20 \%$ de la variabilité interindividuelle du microbiome [4].

Routy et ses collègues ont montré, chez 249 patients atteints de cancer du poumon non à petites cellules, de carcinome rénal ou de cancer de la vessie, que la prise d'antibiotiques (ATB) dans la période de deux mois précédant l'immunothérapie et dans le mois suivant, diminuait la réponse aux $\mathrm{ICl}$. La survie sans progression décroît (3,5 versus 4,1 mois) ( $p=0,017$ ) et la survie globale est plus courte chez les patients ayant pris des ATB ( 11,5 versus 20,6 mois) ( $p<$ $0,001)$ [5]. Cette association négative a été confirmée et approfondie dans une étude récente qui a inclus 239 patients atteints de cancer du poumon et 121 traités pour un cancer du rein [6]. Cette même observation a été notée dans plusieurs modèles murins tumoraux traités avec des $\mathrm{ICl}$ et des ATB à large spectre. De façon intéressante, l'impact des ATB sur le microbiote ne semble pas être limité aux traitements par $\mathrm{ICl}$. Chez les patients recevant une greffe de moelle osseuse, la prise d'ATB peut en effet être associée à une maladie du greffon contre l'hôte (GvHd) sévère au niveau de l'intestin, mais également à une plus faible survie globale $[5,7]$.

Ces résultats suggèrent que la perturbation de la composition du microbiote intestinal et l'absence de certaines espèces de bactéries spécifiques peut 


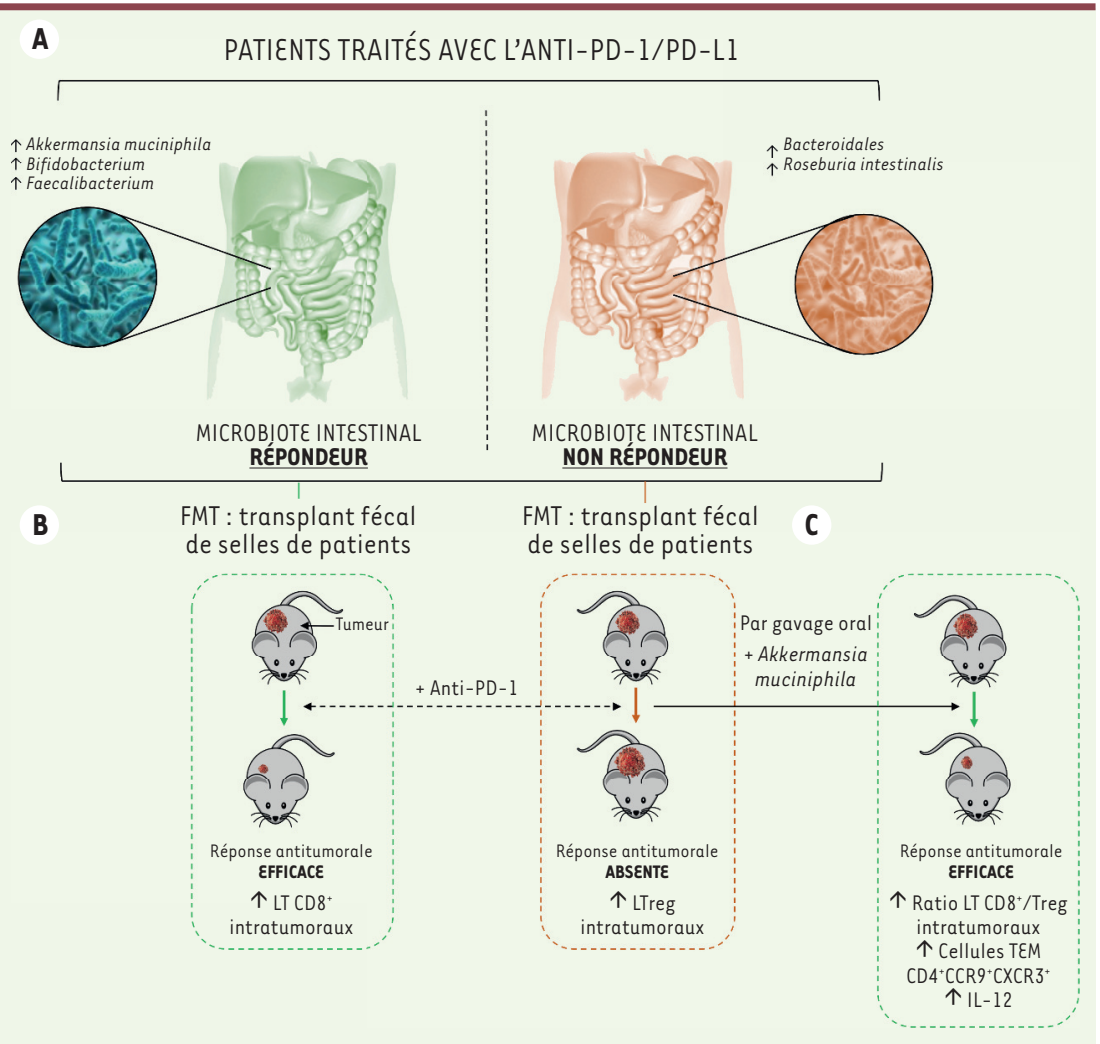

Figure 1. La composition du microbiote intestinal influence la réponse clinique aux inhibiteurs de point de contrôles immunitaires (ICI). A. L'enrichissement de bactéries spécifiques est corrélé à la réponse clinique aux ICI. B. Les transplantations du microbiote fécal (FMT) avec des selles de patients répondeurs chez des souris porteuses de tumeur améliorent la réponse aux anti-PD-1/PD-LI (programmed death-1/PD-ligand 1) avec une augmentation des cellules immunitaires effectrices. Inversement les souris recevant une FMT avec des selles de patients non-répondeurs répondent beaucoup plus faiblement et une augmentation des cellules immunosuppressives $T$ régulatrices (Treg) dans le microenvironnement tumoral est observée. $C$. La compensation des selles de non-répondeurs avec la bactérie Akkermansia muciniphila, par gavage oral, restaure la réponse aux anti-PD-1 chez les souris porteuses de tumeur avec une augmentation des réponses anti-tumorales. LT : lymphocyte T; LTreg : lymphocyte T régulateur ; TEM : cellule T mémoire effectrice ; IL-12 : interleukine-12.

interférer avec l'efficacité des traitements anticancéreux. II a en effet été démontré dans des modèles où des souris porteuses de tumeurs ont été traitées par des anti-CTLA-4 ou anti-PD-Ll que Bacteroides fragilis et Bifidobacterium stimulaient, respectivement, les lymphocytes Thl ( $T$ helper- 1 ) et la maturation des cellules dendritiques anti-tumorales. La présence de la bactérie Bifidobacterium induit de plus une production accrue d'IFN $\gamma$ (interféron- $\gamma$ ) dans les ganglions drainant la tumeur et dans la rate chez les souris traitées par anti-PD-LI [8, 9]. Récemment, par la technique de séquençage métagénomique ou séquençage de I'ARN ribosomal 16S, trois équipes ont indépendamment montré que les patients atteints de cancer du poumon non à petites cellules, de carcinome rénal ou de mélanome métastatique pouvaient être stratifiés par les critères RECIST ${ }^{2}$ version 1.1 en « répondeurs » et «non-répondeurs » aux $\mathrm{ICl}$ selon la composition de leur microbiote intestinal et l'abondance de certaines bactéries [5, 10, 11]. La comparaison du microbiote intestinal par le séquen-

\footnotetext{
${ }^{2}$ Critères de la réponse tumorale en imagerie (response evaluation criteria in solid tumors) fondée sur la mesure du plus grand diamètre des lésions tumorales.
}

çage métagénomique a révélé qu'une augmentation de l'abondance relative d'Akkermansia muciniphila dans une cohorte de 153 patients atteints de cancer du poumon non à petites cellules ou de carcinome rénal était associée à une évolution clinique favorable en réponse au traitement anti-PD-1 [5].

Dans le but d'identifier les bactéries associées à la réactivité aux $\mathrm{ICl}$, Gopalakrishnan et ses collègues ont également étudié le microbiote intestinal et oral de patients atteints de mélanome métastatique traités par des anti-PD-1. De manière intéressante, les patients répondant aux antiPD-1 présentaient une abondance relative élevée dans leur microbiote intestinal de bactéries du genre Faecalibacterium, alors que les bactéries de l'ordre des Bacteroidales étaient abondantes chez les patients non-répondeurs [10].

Aucune corrélation microbienne n'est observée avec le microbiote oral, ce qui suggère que seule la composante bactérienne intestinale est la source d'une synergie bactério-immunitaire pour la réponse aux anti-PD-1. Matson et ses collègues ont analysé le microbiote intestinal de patients, atteints d'un mélanome métastatique, traités par un anti-PD-Ll et révèlent une augmentation de huit espèces microbiennes dont Bifidobacterium longum. Chez la souris, ils ont de plus montré que la présence de cette espèce dans les intestins d'animaux porteurs d'une tumeur améliorait la thérapie par des anti-PD-Ll [11].

Pour approfondir ces résultats, des transplantations de microbiote fécal (FMT) ont été réalisées en utilisant des selles de patients répondeurs et non-répondeurs chez des souris axéniques (dépourvues de microbiote et vivant dans des isolateurs) ou recevant des ATB, porteuses de tumeurs et traitées par antiPD-1 ou anti-PD-Ll (Figure 1) $[5,10$, $11,12](\rightarrow)$. Les études menées

$(\rightarrow)$ Voir la Synthèse de J.C. Lagier et $\mathbf{D}$. Raoult, $m / s n^{\circ} 11$, novembre 2016, page 991

par Routy et Gopalakrishnan ont décrit une meilleure réponse aux anti-PD-1 chez les souris recevant une FMT de patients 
répondeurs que chez les souris colonisées avec des fèces provenant de patients non-répondeurs. Gopalakrishnan et ses collègues ont également montré que cette réponse favorable était corrélée avec une plus grande abondance de Faecalibacterium dans les fèces des souris [10].

Matson et ses collègues ont, quant à eux, observé que le traitement par des antiPD-Ll était efficace chez les souris recevant une FMT de patients comprenant cinq des huit souches initialement associées à la réponse aux anti-PD-Ll chez l'homme, parmi lesquelles Bifidobacterium [11]. De manière intéressante, l'étude de Matson montre également que les souris recevant une FMT avec des fèces de patients répondeurs possédaient une densité plus élevée de LT CD8 ${ }^{+}$intratumoraux spécifiques de la tumeur avec une baisse des LT régulateurs Fox $\mathrm{P3}^{+} \mathrm{CD}^{+3}$ en comparaison aux souris qui ont reçu une FMT avec des fèces de patients non-répondeurs [11]. De plus, un infiltrat plus important de cellules $\mathrm{CD}^{+}$a également été observé dans des biopsies de patients atteints de mélanome répondeurs comparés aux nonrépondeurs. Les $\mathrm{ICl}$ ciblent donc le répertoire et l'activité des cellules immunitaires de l'hôte et induisent des réponses antitumorales effectrices efficaces.

Une question importante et cliniquement pertinente est de savoir si la manipulation du microbiote intestinal pourrait transformer les patients non-répondeurs aux $\mathrm{ICl}$ en répondeurs. Notre groupe a abordé cette question en modulant le microbiote des souris recevant une FMT avec des selles de patients non-répondeurs par un gavage additionnel avec des bactéries Akkermansia muciniphila (Figure 1). Cette compensation du microbiote a entraîné une augmentation de l'efficacité des anti-PD-1 et induit une activation des cellules dendritiques produisant de l'interleukine-12 qui a provoqué la mobilisation des $\mathrm{LT} C D 4^{+} \mathrm{CCR}^{+}$( $C-C$ motif chemokine receptor 9$)^{4}$ du ganglion mésentérique vers les ganglions drainants

\footnotetext{
3 Sous-population de lymphocytes $\mathrm{T} \mathrm{CD} 4^{+}$régulateurs qui
} expriment le facteur de transcription FoxP3 (forkhead box P3). la tumeur ainsi que dans le site tumoral. Modifier le microbiote peut donc moduler le système immunitaire et la réponse $T$ anti-tumorale spécifique.

Les résultats conjoints de ces trois études suggèrent que le microbiote des patients répondeurs contient des bactéries immunogéniques telles que $A$. muciniphila, Faecalibacterium et $B$. longum, qui induisent une efficacité fonctionnelle des anti-PD-1/PD-Ll (Figure 1). Un nouveau concept thérapeutique émerge qui suggère que l'efficacité des $\mathrm{ICl}$ pourrait être améliorée par la modulation du microbiote intestinal. De plus, dans ces trois études d'autres bactéries telles que Clostridiales, Enterococcus fecium, Eubacterium spp., Firmicutes et Ruminococcus spp. ont également été associées aux répondeurs, mais à des degrés moindres $[5,10,11]$.

Chez des patients non-répondeurs, plusieurs stratégies thérapeutiques peuvent être envisagées en combinaison avec les $\mathrm{ICl}$ telles que: (1) des transplantations du microbiote fécal de selles de patients répondeurs afin d'augmenter la diversité bactérienne et de complémenter leur microbiote; (2) l'administration de prébiotiques et/ou de probiotiques. Les prébiotiques regroupent des nutriments qui vont permettre la prolifération de certaines bactéries du microbiote intestinal. Une étude récente a en effet montré que l'administration d'un prébiotique, l'extrait de canneberge (ou grande airelle rouge), à des souris obèses permettait d'augmenter le taux d'A. muciniphila dans leur microbiote intestinal et diminuait l'insulinorésistance et la stéatose hépatique [13]. Des traitements probiotiques des cancers, ou oncomicrobiotiques, consisteraient à administrer des bactéries encapsulées, vivantes ou atténuées, spécifiques du phénotype de répondeurs à des patients non-répondeurs.

De nombreux aspects restent toutefois à préciser, notamment le volet mécanistique afin d'expliquer l'augmentation d'activité des cellules effectrices du système immunitaire par ce groupe de bactéries. Le microbiote pourrait ainsi être considéré comme une nouvelle cible en immuno-oncologie ouvrant très prochainement de nouvelles perspectives pronostiques et thérapeutiques aux patients. $\diamond$ In bacteria veritas: pronostic role of intestinal microbiote in cancer therapy

\section{LIENS D'INTÉREิT}

Les auteurs déclarent n'avoir aucun lien d'intérêt concernant les données publiées dans cet article.

\section{RÉFÉRENCES}

1. Marabelle A, Routy B, Michels J, et al. Prime time for immune-checkpoint targeted therapy at ASCO 2015. Oncoimmunology 2016 ; 5 : e1068494.

2. Gandhi L, Rodríguez-Abreu D, Gadgeel S, et al. Pembrolizumab plus chemotherapy in metastatic non-small-cell lung Cancer. $N$ Engl J Med 2018. doi: 10.1056/NEJMoal801005

3. Patel SA, Minn AJ. Combination cancer therapy with immune checkpoint blockade: mechanisms and strategies. Immunity $2018 ; 48: 417-33$.

4. Rothschild D, Weissbrod 0, Barkan $\varepsilon$, et al. Environment dominates over host genetics in shaping human gut microbiota. Nature 2018 ; 555 : 210-5.

5. Routy B, Le Chatelier $\varepsilon$, Derosa L, et al. Gut microbiome influences efficacy of PD-1-based immunotherapy against epithelial tumors. Science $2018 ; 359: 91-7$.

6. Derosa L, Hellmann MD, Spaziano M, et al. Negative association of antibiotics on clinical activity of immune checkpoint inhibitors in patients with advanced renal cell and non-small cell lung cancer. Ann Oncol 2018 ; Mar 30. doi: 10.1093/annonc/ mdy 103 .

7. Shono Y, Docampo MD, Peled JU, et al. Increased GVHD-related mortality with broad-spectrum antibiotic use after allogeneic hematopoietic stem cell transplantation in human patients and mice. Sci Transl Med 2016; 8 : 339ra71.

8. Vétizou M, Pitt JM, Daillère R, et al. Anticancer immunotherapy by CTLA-4 blockade relies on the gut microbiota. Science 2015 ; 350 : 1079-84.

9. Sivan A, Corrales L, Hubert N, et al. Commensal Bifidobacterium promotes antitumor immunity and facilitates anti-PD-Ll efficacy. Science 2015; 350 1084-9.

10. Gopalakrishnan V, Spencer CN, Nezi L, et al. Gut microbiome modulates response to anti-PD-1 immunotherapy in melanoma patients. Science 2018 359: 97-103.

11. Matson V, Fessler J, Bao R, et al. The commensal microbiome is associated with anti-PD-1 efficacy in metastatic melanoma patients. Science 2018 ; 359 : 104-8.

12. Lagier JC, Raoult D. Greffe de microbiote fécal et infections : mise au point, perspectives. Med Sci (Paris) $2016 ; 32: 991-7$.

13. Anhê FF, Nachbar RT, Varin TV, et al. A polyphenol-rich cranberry extract reverses insulin resistance and hepatic steatosis independently of body weight loss. Mol Metab 2017 ; 6 : 1563-73. 